\title{
Capsular fibrosis: a review of prevention methods and management
}

\author{
Andreea D. Fișuș ${ }^{1,2} \cdot$ Oliver Findl $^{1}$
}

Received: 30 September 2019 / Revised: 27 October 2019 / Accepted: 13 November 2019 / Published online: 5 December 2019

(c) The Royal College of Ophthalmologists 2019

\begin{abstract}
Opacification of the posterior capsule caused by residual lens epithelial cells (LEC) is still the most frequent long-term complication of cataract surgery. Beside the opacification of the visual axis with posterior capsule opacification (PCO), resulting in a decrease in visual function, fibrotic changes may also have a mechanical effect on intraocular lens (IOL) position such as axial shift, decentration, tilt and capsule striae. In this article, two types of capsular fibrosis are explored, on the one hand the anterior capsule fibrosis and on the other hand PCO. Results from clinical trials concerning their causes, natural course, incidence, influencing factors and possible methods of prophylaxis are presented.
\end{abstract}

\section{Introduction}

Posterior capsule opacification (PCO) is still a major longterm complication of cataract surgery and is a leading cause of non-refractive visual decrease [1-5].

PCO is relevant because it results in a decrease in patient satisfaction after surgery, its treatment has potential complications, as well as an increased necessity for long-term follow-up and the socioeconomical impact [3, 6-10].

\section{Incidence}

The incidence of PCO is estimated at $11.8 \%$ at 1 year and $18.4-8.4 \%$ at 5 years after cataract surgery $[2,11,12]$.

Some studies have shown that diabetic patients have higher PCO rates than non-diabetic [13]. A study conducted by Praveen et al. showed similar degrees of PCO at 4 years of follow-up in cases of diabetic and non-diabetic patients, concluding that differences exist only in the early postoperative period, due to a decrease of density of LEC in

Oliver Findl

oliver@findl.at

1 Department of Ophthamology, Vienna Institute for Research in Ocular Surgery - Karl Landsteiner Institute, Hanusch Hospital, Vienna, Austria

2 George Emil Palade University of Medicine, Pharmacy, Science and Technology of Târgu-Mureș, Târgu-Mureș, România diabetic capsular bags [14]. The duration and severity of diabetes may play a role [14].

When looking into lens factors and PCO incidence, studies placed the percentage of this complication at $4.9 \%$ (24\% at a 5 year retrospective study) [3] in case of hydrophobic intraocular lenses (IOLs) and 50.3\% for hydrophilic IOLs [2]. The IOL material plays a role on facilitating a capsular-lens adhesion, thus helping in the creation of a capsular bend $[1,7,15,16]$.

\section{Aetiology}

PCO is known as being a multifactorial pathology [1]. Studies shown that patient-related factors influence PCO incidence, such and age, sex and ocular comorbidities. Nevertheless, the relative contribution of these factors in relation to IOL factors is not known [2]. PCO is induced by LEC that undergo an EMT, proliferating and migrating to the posterior capsule $[8,11]$. It is not known whether individual patient factors that promote PCO development are influenced by intrinsic proprieties of the LEC, or due to differences in the ocular milieu surrounding the LEC in vivo [2].

\section{Pathogenesis}

\section{Lens capsule and lens epithelial cells}

The lens capsule consists of an anterior surface, that has a single row of LEC, an equatorial region and a posterior 
capsule, that consists only of fibres [11]. The anterior surface is considered to be quiescent, while in the equatorial region, considered the germinative zone, have the LEC that are able to proliferate $[11,17]$.

The typical response of the LEC of the anterior capsule, also named a-cells, is to proliferate into fibrous-like tissue, undergoing a process of metaplasia $[17,18]$. The LEC of the equatorial region are considered to be pluripotent [17]. The equatorial LEC undergo mitosis and continuously produce new lens fibres, also resulting in Soemerring ring formation late after surgery [17]. Nevertheless, the possible pluripotency of the anterior LEC as well as the clear separation of these two subtypes of LEC is still in discussion [11].

LEC have the ability, under in vitro conditions such as in cells culture, to proliferate and migrate to the other side of the anterior capsule [11]. Andjeli et al. showed that the LEC have the ability to migrate to the posterior capsule in an interval of 1-2 weeks after surgery [11]. The role of the LEC is to keep the internal milieu of the lens, in order to perform this role its integrity is important [19].

\section{Posterior capsule opacification development}

It is known that PCO is a response in cellular healing and clinically two types of PCO may be found between the IOL and posterior capsule, both having cyst-like structure [20].

The first of these types of PCO has been described as regeneratory, with lens like material filling the space between IOL and posterior capsule containing Elsching pearls, being a cluster of swollen cells [17, 21, 22]. These are thought to derived from the pre-equatorial region of the capsule. The other type is the fibrotic type, thought to originate from the residing LEC of the anterior capsule [4, 17, 20, 22-24]. PCO can either be predominantly the one or the other type or a combination of the two [17, 23].

The development of PCO is variable with a time frame between few months to 4 years after surgery [17]. Although initially it was considered that Elschnig pearls would develop gradually and slowly, studies showed that these undergo a rapid turnover within days and weeks $[20,25]$.

Fig. 1 Changes of PCO morphology during a 4-week period; after 2 weeks large pearl disappear by Buehl et al. [24].
Neumayer et al. tried to identify individual pearls from consecutive images taken over a period of 3 years and concluded that the formation of pearls is a dynamic process [26].

A better understanding of the course of PCO formation is important in developing possible pharmacological means of LEC modulation [20]. Findl et al. showed that the changes in the number, formation and decrease in size of Elschnig pearls is heterogeneous [20]. Some eyes presented with high turnover of pearls, while others presented with little change [20, 24, 26, 27] (Fig. 1). The same authors described large variability of pearl size over time, postulating that they do not necessarily disappear completely, just become small and are more difficult to be seen in retroillumination photography and found no correlation to the patient factors or IOL specifications [20].

A possible explanation for a reduction of laser capsulotomy rate 4 years after surgery is that the pearls lose their capacity to proliferate and LEC may be undergoing apoptosis $[26,28]$. Apoptosis of these cells may also be obtained by posterior capsule opening which leads to cytokines from the aqueous having access to LEC [26]. Interestingly, Elschnig pearls that disappeared, or were "newly" formed were usually smooth in shape and quite round [20].

\section{Epithelial-mesenchymal transition}

It is known that after trauma or surgery, various cytokines and growth factors levels increase in the aqueous humour stimulating the LEC to undergo epithelial-mesenchymal transition (EMT), thus proliferating [29-35]. Inhibiting LEC proliferation is a feasible method in attempting to reduce PCO [29, 35].

The EMT characteristics include the transformation into spindle-shaped cell that is followed by the accumulation of $\alpha$-smooth muscle actin ( $\alpha \mathrm{SMA})$ and the loss of epithelial markers [31, 35].

Due to the surgical trauma during formation of the capsulorhexis, transforming growth factor (TGF $\beta$ ) is released and through the Smad signalling pathway the EMT process is induced [13, 31, 33-35]. TGF $\beta$ has the role of inducing the expression of $\alpha \mathrm{SMA}$, fibronectin and collagen type I
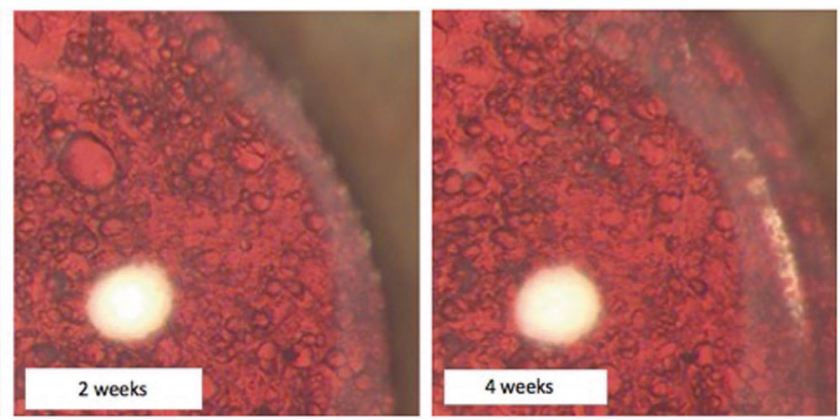
and III and reduces the expression of vimentin, Snail1/2 and E-cadherin, these changes are the characteristic of EMT $[13,30-32,36]$. Knowing that the degree of LEC fibrosis is linked to quality and quantity by collagen synthesis, Nishi et al. detected the synthesis of collagen I, IV, V and VI in LEC cultures [18].

Sudelin et al. looked at the differences between patient that underwent Neodymium-doped yttrium aluminium garnet (Nd:YAG) capsulotomy in a period of 5 years after cataract surgery and found no significant differences in cell growth between patient that underwent treatment and those who did not, concluding that the inherent proliferation capacity of lens epithelial cells (LEC) is not a major contributor in PCO development [2]. The proliferation of cells is linked to the local milieu, such as the presence of growth factors, capsule configuration and IOL material more than individual factors [2].

Concerning outcome measures of $\mathrm{PCO}$, the rate of $\mathrm{Nd}$ : YAG laser capsulotomy is not an accurate since it is influenced by various factors such as patient demand as well as financial incentives of the physician [14].

Raghavan et al. showed that LEC of young patients react poorly to TGF $\beta$ and consequently synthesise lower levels of $\mathrm{SMA}$ and this weaker reaction is linked to young cells ability of regenerating fibre cells [13].

It is frequently reported that anterior LEC do not undergo mitosis, however, studies shown they have this property under different conditions [11]. By demonstrating the presence of Sox 2 in cells cultures of LEC derived from anterior capsules, shows that the anterior LEC are pluripotent and can proliferate [11].

\section{Prophylaxis}

The methods of prevention may be summarised into two categories: reducing the number of LEC remaining in the capsular bag and creating a barrier to their migration to the posterior capsule [17]. Some studies have shown that the presence of at least some residual LEC after surgery may be beneficiary in order to keep the capsule vital [35].

There are six steps known to increase the chances of delaying PCO formation: a good cortical clean-up, an in the bag fixation of the lens, performing a capsulorhexis that is smaller than the optic diameter, thus creating a tight adhesion between the capsule and IOL, known as a shrink wrap, created also by the use of IOLs known to have higher bioadhesiveness and a maximal contact between the capsule and the IOL and the use of IOL with a square optic geometry creating an effective migration barrier [15, 17, 37]. Although it is difficult to assess the influence of each of these factors in reducing laser capsulotomies, multiple studies point at the IOL having a significant influence compared with other factors. Concerning the IOL characteristics, a major role is played by the optic edge $[1,2,4,37]$.

\section{IOL material and design}

IOL characteristics and proper surgical technique stand out as important factors in reducing PCO incidence [3]. The IOL characteristics implied in PCO prevention may be divided into IOL design and IOL material. Multiple studies have shown that hydrophobic acrylic IOL with sharp-edge optic have resulted in significantly lower capsulotomy rates $[3,5,7,9,16]$.

\section{IOL material}

In vitro and in vivo studies, demonstrated that cells migration under acrylic material is lower than with PMMA IOLs [4, 38, 39]. Acrylic hydrophobic material are known to have higher adhesion capacity and thus more rapid capsular bend formation, limiting the space into which LEC can migrate $[1,5,16,38,39]$.

A study conducted by Sacu et al. found the comparable amounts of PCO when comparing silicone to acrylic and PMMA IOLs, nevertheless the silicone type lenses presented with more anterior capsule opacification (ACO), shown as whitening of the rhexis edge as well as capsule contraction, regardless of optic edge design [40].

A Cochrane review showed hydrophilic acrylic had higher PCO rates than hydrophobic materials [4]. However, one major confounding factor was that hydrophilic IOLs often have less sharp optic edges, so that the material effect may be much less than shown in trials comparing hydrophobic to hydrophilic acrylic IOL.

\section{IOL design}

A number of clinical studies demonstrated the lower incidence of PCO, both regeneratory and fibrotic, in case of a sharp truncated optic edge and especially sharp posterior optic edge of the IOL, when compared with round optic edge IOL [5, 25, 40]. Also, in vitro studies demonstrated the effective role of the square optic edge, although contrary results have been obtained with continues and interrupted square edge designs $[5,9,38]$.

The role of the square edge resides on a physical barrier to LEC migration $[2,4,5,17,24,25]$. Although this edge design showed superiority compared with other IOL factors, the barrier may be overrun with time [2, 5, 37, 38, 41]. A $360^{\circ}$ overlapping of the anterior capsule rhexis edge with the IOL optic appears to be important in insuring the positive effect of the sharp-edge IOL $[1,15]$.

A more bulky haptic-optic junction will not allow sufficient capsule bending at the sharp optic edge allowing 
LEC to migrate to the posterior capsule $[1,7,38,41]$. Therefore, a three-piece IOL design with thin haptics allowing complete fusion of the anterior and posterior capsule all the way around the optic edge and a better capsule bend formation may have a better PCO protective effect than single-piece designs $[1,7,38]$. A thinner haptic may also aid in a more effective adhesion between the anterior and posterior capsule in the area of the haptic-optic junction, thus enhancing the effect of the sharp edge of the optic [7]. There is a clear trend that PCO will invade and cross the barrier close to the optic-haptic junction due to this failure of causing a permanent bending of the posterior capsule at the optic edge [42] (Fig. 2).

Studies have shown conflicting results in difference of $\mathrm{ACO}$ and PCO development when one piece or three-piece lenses were used [1, 38]. Sacu et al. reported significant differences in PCO rates at 1 year between a three-piece and one piece IOL, however the result at 2 years were no longer significant statistically [40, 43]. Some studies showed a lower incidence of capsular fibrosis when $6.0 \mathrm{~mm}$ diameter IOL were implanted as opposed to $5.5 \mathrm{~mm}$ [7]. In general, however, even though better concerning PCO prevention, three-piece IOLs are used less commonly due to the difficulties in using these in preloaded injector systems and the higher costs in production.

Buehl et al. showed that the optic edge plays a more important role than the IOL material with a square edge significantly reducing the incidence of $\mathrm{PCO}$ at a 3 year follow-up, where the only difference between the IOLs was the optic edge, both lenses used in the study being acrylic hydrophobic [24]. One drawback was positive dysphotopsia such as glare created by the sharp optic edge [24]. Studies have shown similar PCO reducing effects when using PMMA material, sharp-edge optic being superior than round edge [6].

Sacu et al. developed a classification system for the interaction of the anterior and posterior capsules and the optic edge of the IOL and assessed these for a series of IOL differing in haptic and optic edge design as well as optic material [40]. The authors found that the most permanent barrier to LEC migration appears to be a shrink-wrapping configuration, where the posterior capsule is pulled slightly onto the anterior IOL optic surface due to the extensive fibrosis of the anterior capsule [40]. This is found especially with silicone IOL optic material and may result in the most permanent barrier formation since it is difficult for late regenerating LEC from the capsule bag equator to re-open the fused capsule bag leaflets at the optic edge [42] (Fig. 3). However, silicone material is used less commonly for IOLs due to difficulties in easy and safe implantation with preloaded injectors which are used more and more readily in surgery.

\section{Surgical technique}

The quality of surgery is linked strongly to the formation of PCO [17]. Surgical factors known to decrease PCO incidence are thorough cortical clean-up, capsular polishing, IOL implantation in the bag and use of a continuous curvilinear capsulorhexis $[6,7,15,17,40]$.

Anterior capsule polishing has been shown to reduce ACO [25]. However, since fewer LEC remain near the rhexis edge and due to the lower degree of ACO, the
Fig. 2 Comparison of barrier effect loss between silicon $(\mathbf{a}-\mathbf{c})$ and hydrophobic acrylic IOLs $(\mathbf{d}-\mathbf{f})$ at $1(\mathbf{a}, \mathbf{d}), 3(\mathbf{b}, \mathbf{e})$ and 6 (c, f) years after surgery by Vock et al. [42].

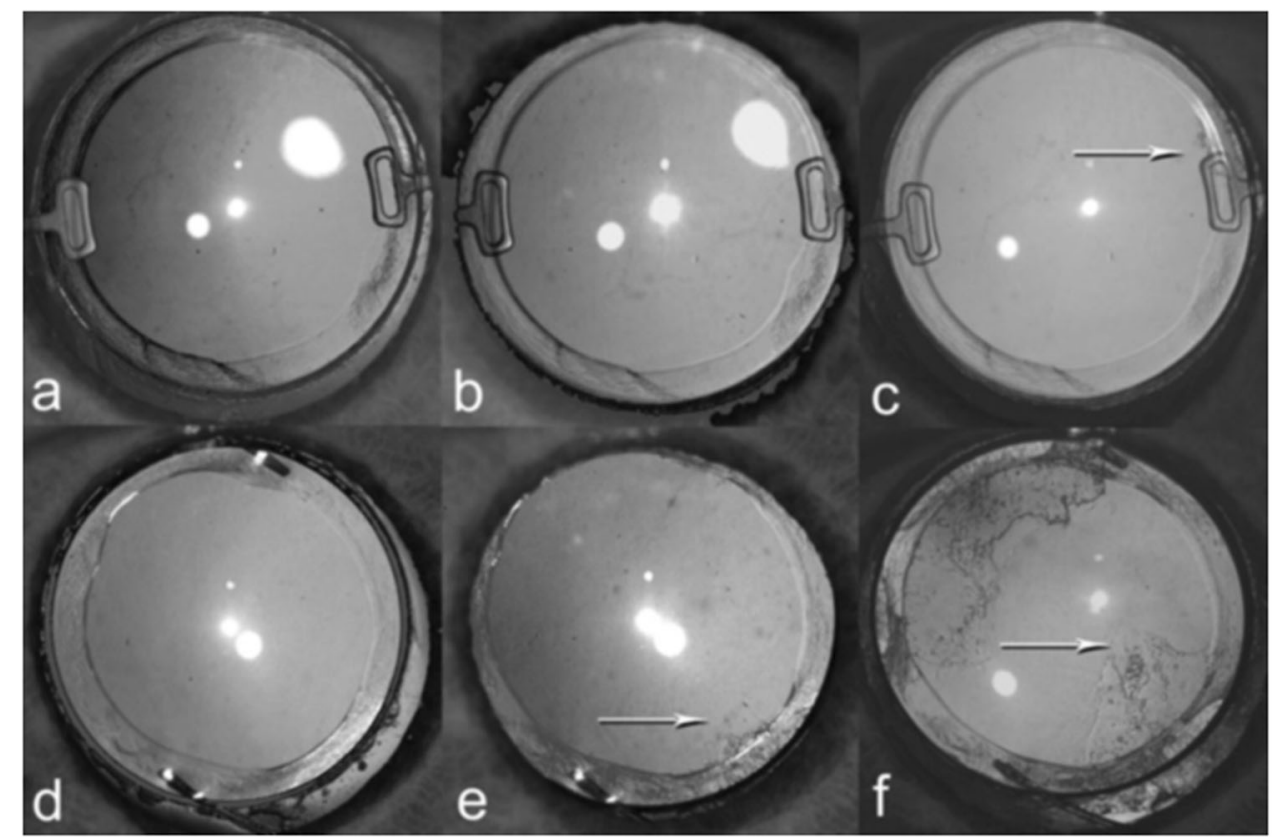




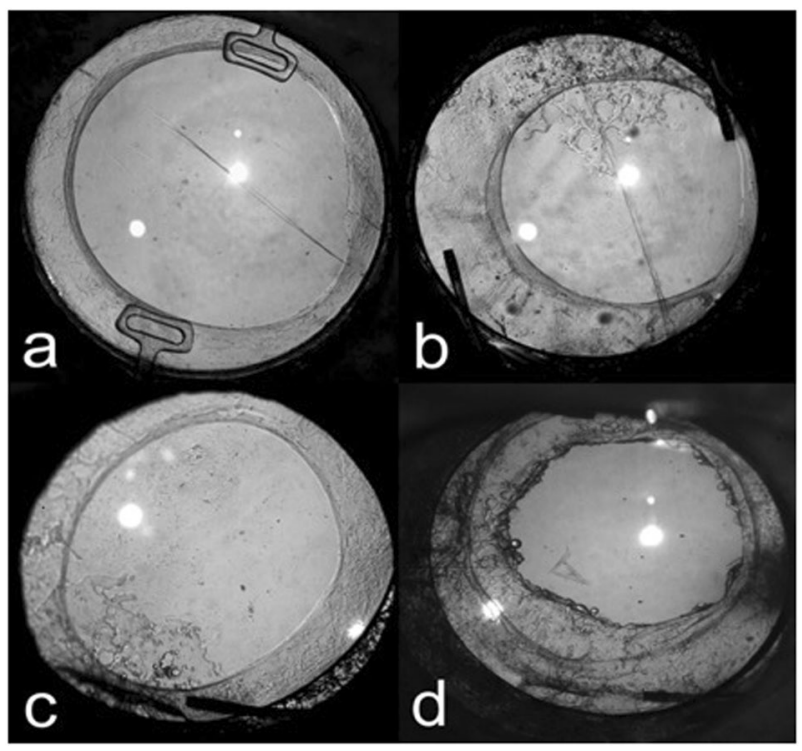

Fig. 3 Location of sharp-edge optic barrier failure: no barrier loss (a), inside the inner curvature of the haptic loop (b), outside the inner curvature of the haptic loop (c) and not discernable (d) by Vock et al. [42].

regeneratory LEC that invade later after surgery can more easily re-open the capsule bag due to the lack capsule-lens adhesion and this results in a loss of capsule bending at the sharp optic edge resulting in PCO formation [25]. Thus, anterior capsular polishing appears to not be beneficiary in reducing the incidence of $\mathrm{PCO}$, but actually to enhance PCO formation [25].

\section{Treatment}

Drugs that act on cell receptors, cell adhesion molecules and their ligands have been investigated, however due to their cell toxicity, they have not been used in clinical trials [12]. Studies that investigated cancer drugs, such as cinobufacini, have shown their role in inducing LEC apoptosis in a dosedependent fashion [12]. Since the proliferation of LEC is linked to the dynamics of the actin cytoskeleton, studies have shown that cytoskeletal drugs ( $\mathrm{H} 7$, a broad-spectrum serine-theonine kinase inhibitor and Latruculin B) may influence PCO formation in cell cultures, however, a single dose may not be sufficient for the prevention of PCO [44].

Another study looked at the effect of distilled water and hydrogen peroxide, concluding it had a delaying effect on PCO, nevertheless, not preventing EMT [45].

Pharmacological methods that are cytotoxic have not been used clinically due to concerns of collateral damage to the surrounding tissue, although aqueous humour circulation may diminish the drug concentration rather quickly [44].
A novel approach may be the use of coating IOL with cytotoxic drugs that ideally result in no damage to surrounding tissues [46].

\section{PCO evaluation systems}

To date many PCO evaluation systems have been developed. One option is subjective grading at the slit lamp or from retroillumination photographs. Such a subjective grading system, evaluation of posterior capsular opacification, was introduced by Tetz et al. A more quantitative grading of $\mathrm{PCO}$, assessed the area of $\mathrm{PCO}$ behind the IOL optic. A more user-independent quantitative method is the AQUA method, automated quantification of after cataract [23].

\section{Anterior capsule opacification}

After capsulorhexis the anterior capsule is in direct contact with the IOL, the remaining LEC have the potential to undergo proliferation and create ACO [17, 47].

Anterior capsular shrinkage and constriction of the capsular opening may be accompanied by an anterior capsule fibrosis, which may lead to capsular phimosis, excessive zonular traction which in turn may lead to IOL decentration or dislocation [17, 47].

ACO appears to form in two stages, an early stage consisting of cell proliferation and a late stage which consists of cell degeneration or LEC disappearance [17]. Factors linked to ACO development are the size of the capsulorhexis with smaller size having a positive effect, and IOL material and design. Silicone lenses are associated with capsular shrinkage and acrylic IOL with less ACO [17]. Performing a larger capsulorhexis may avoid contraction of the capsule especially in eyes at risk such as those with pseudoexfoliation syndrome [14].

\section{Conclusion}

LEC migration appears to be inhibited by IOL with high bioadesiveness, acrylic hydrophobic material and a sharp optic edge design. The focus of PCO research should be on modulating LEC with pharmacological means, possibly adherent to the IOL material.

\section{Compliance with ethical standards}

Conflict of interest $\mathrm{OF}$ is a scientific advisor to Alcon, Carl Zeiss Meditec AG, Johnson \& Johnson and Merck. The other author declares no conflict of interest. 
Publisher's note Springer Nature remains neutral with regard to jurisdictional claims in published maps and institutional affiliations.

\section{References}

1. Zemaitiene R, Vytautas J, Auffarth GU. Influence of three-piece and single-piece designs of two sharp-edge optic hydrophobic acrylic intraocular lenses on the prevention of posterior capsule opacification: a prospective, randomised, long-term clinical trial. Br J Ophthalmol. 2007;91:644-8.

2. Sundelin K, Almarzouki N, Soltanpour Y, Petersen A, Zetterberg M. Five-year incidence of Nd:YAG laser capsulotomy and association with in vitro proliferation of lens epithelial cells from individual specimens: a case control study. BMC Ophthalmol. 2014;14:116-23.

3. Johansson B. Clinical consequences of acrylic intraocular lens material and design: Nd:YAG-laser capsulotomy rates in $3 \times 300$ eyes 5 years after phacoemulsification. Br J Ophthalmol. 2010;94:450-5.

4. Findl O, Buehl W, Bauer P, Sycha T. Interventions for preventing posterior capsule opacification. Cochrane Database Syst Rev. 2007. https://doi.org/10.1002/14651858.CD003738.pub2.

5. Schartmuller D, Schiefl S, Schwarzenbacher L, Leydolt C, Kundi M, Pieh S, et al. Posterior capsule opacification and Nd:YAG laser rates with two hydrophobic acrylic single-piece IOLs. Eye. 2019. https://doi.org/10.1038/s41433-019-0569-x.

6. Hazra S, Palui H, Vemuganti GK. Comparison of design of intraocular lens versus the material for PCO prevention. Int $\mathbf{J}$ Ophthalmol 2012;5:59-63.

7. Mian SI, Fahim K, Marcovitch A, Gada H, Musch DC, Sugar A. Nd:YAG capsulotomy rates after use of the AcrySof acrylic three piece and one piece intraocular lenses. Br J Ophthalmol 2005; 89:1453-7.

8. Wormstone IM, Eldred JA. Experimental models for posterior capsule opacification research. Exp Eye Res. 2016;142:2-12.

9. Ursell PG, Dhariwal M, Majirska K, Ender F, Kalson-Ray S, Venerus A, et al. Three-year incidence of Nd:YAG capsulotomy and posterior capsule opacification and its relationship to monofocal acrylic IOL biomaterial: a UK real world evidence study. Eye. 2018;32:1579-89.

10. Aaronson A, Grzybowski A, Tuuminen R. The health economic impact of posterior capsule opacification in Findland comparing the two single-piece intraocular lenses: a cost-consequence analysis. Acta Ophthalmol. 2019. https://doi.org/10.1111/aos.14139.

11. Andjelic S, Draslar K, Lumi X, Yan X, Graw J, Facko A, et al. Morphological and proliferative studies on ex vivo cultured human anterior lens epithelial cells-relevance to capsular opacification. Acta Ophthalmol. 2015;93:499-506.

12. Xu GX, Wang TT. Apoptosis of lens epithelial cells induced by cinobufagin. Int J Ophthalmol. 2010;3:128-31.

13. Raghavan CT, Smuda M, Smith AJO, Howell S, Smith DG, Singh A, et al. AGEs in human lens capsule promote the TGFß2mediated EMT of lens epithelial cells: implications for ageassociated fibrosis. Aging Cell. 2016;15:465-76.

14. Praveen MR, Vasavada AR, Shah GD, Shah AR, Khamar BM, Dave KH. A prospective evaluation of posterior capsule opacification in eyes with diabetes mellitus: a case-control study. Eye. 2014;28:720-7.

15. Fang Y, Xixia D, Jin L, Lei L, Pingjun C, Hongfang Z, et al. Relationship of posterior capsular opacification and capsular bend type investigation based on swept-source optical coherence tomography. Curr Eye Res. 2019. https://doi.org/10.1080/ 02713683.2019.1645183.

16. Zhao Y, Yang K, Li J, Huang Y, Zhu S. Comparison of hydrophobic and hydrophilic intraocular lens in preventing posterior capsule opacification after cataract surgery an updated metaanalysis. Medicine. 2017;96:44-53.

17. Apple DJ. Influence of intraocular lens material and design on postoperative intracapsular cellular reactivity. Tr Am Ophth Soc. 2000;98:257-83.

18. Nishi O, Nishi K, Fujiwara T, Shirasawa E. Types of collagen synthesised by the lens epithelial cells of human cataracts. Br J Ophthalmol. 1995;79:939-43.

19. Andjelic S, Zupancic G, Perovsek D, Hawlina M. Human anterior lens capsule epithelial cells contraction. Acta Ophthalmol. 2011;89:645-53.

20. Findl O, Neumayer T, Hirnschall N, Buehl W. Natural course of elschnig pearl formation and disappearance. Ophthalmol Vis Sci. 2010;51:1547-53.

21. Eldred JA, Zheng J, Chen S, Wormstone M. An in vitro human lens capsular bag model adopting a graded culture regime to assess putative impact of IOLs on PCO formation. Invest Ophthalmol Vis Sci. 2019;60:113-22.

22. Lu C, Yu S, Song H, Zhao Y, Xie S, Yuan X. Posterior capsular opacification comparison between morphology and objective visual function. BMC Ophthalmol. 2019;19:40-48.

23. Kronschläger M, Siegl H, Pinz A, Feichtenhofer C, Buehl W, Hirnschall N, et al. Automated qualitative and quantitative assessment of posterior capsule opacification by Automated Quantification of After-Cataract II (AQUA II) system. BMC Ophthalmol. 2019; 19:114-20.

24. Buehl W, Findl O, Menapace R, Sacu S, Kriechbaum K, Koeppl C, et al. Long-term effect of optic edge design in an acrylic intraocular lens on posterior capsule opacification. J Cataract Refract Surg. 2005;31:954-61.

25. Sacu S, Menapace R, Findl O, Georgopoulos M, Buehl W, Kriechbaum K, et al. Influence of optic edge design and anterior capsule polishing on posterior capsule fibrosis. J Cataract Refract Surg. 2004;30:658-62.

26. Neumayer T, Findl O, Buehl W, Sacu S, Menapace R, Georgopoulos M. Long-term changes in the morphology of posterior capsule opacification. J Cataract Refract Surg. 2005; 31:2120-8.

27. Buehl W, Findl O, Neumayer T, Georgopoulos M, Sacu S. Shortterm changes in the morphology of posterior capsule opacification. J Cataract Refract Surg. 2005;31:962-8.

28. Caballero A, Marin JM, Salinas M. Spontaneous regression of Elschnig pearl posterior capsule opacification. J Cataract Refract Surg. 2000;26:779-80.

29. Xiao W, Chen X, Li W, Ye S, Wang W, Luo L, et al. Quantitative analysis of injury-induced anterior subcapsular cataract in the mouse: a model of lens epithelial cells proliferation and epithelialmesenchymal transition. Sci Rep. 2015;5:8362 https://doi.org/10. 1038/srep08362

30. Gotoh N, Perdue NR, Matsushima H, Sage EH, Yan Q, Clark JI. An in vitro model of posterior capsular opacity: SPARC and TGFB2 minimize epithelial-to-mesenchymal transition in lens epithelium. Invest Ophthalmol Vis Sci. 2007;48:4679-87.

31. Eldred JA, Dawes LJ, Wormstone IM. The lens as a model for fibrotic disease. Philos Trans R Soc B. 2011;366:1301-19.

32. Liu L, Xiao W. Notch1 signalling induces epithelial-mesenchymal transition in lens epithelium cells during hypoxia. BMC. 2017; 17:135-42.

33. Taiyab A, Korol A, Deschamps PA, West-May JA. $\beta$-Catenin/ CBP-dependent signalling regulates TGF- $\beta$-induced epithelial to mesenchymal transition of lens epithelial cells. Invest Ophthalmol Vis Sci. 2016;57:5736-47.

34. Jiang J, Shihan MH, Wang Y, Duncan MK. Lens epithelial cells initiate an inflammatory response following cataract surgery. Invest Ophthalmol Vis Sci. 2018;59:4986-97. 
35. Lovicu FJ, Shin EHH, Mcavoy JW. Fibrosis in the lens. Sprouty regulation of TGFß-signaling prevents lens EMT leading to cataract. Exp Eye Res. 2016;142:92-101.

36. Eldred JA, McDonald M, Wilkes HS, Spalton DJ, Wormstone IM. Growth factor restriction impedes progression of wound healing following cataract surgery: identification of VEGF as a putative therapeutic target. Sci Rep. 2016;6:24453 https://doi.org/10.1038/ srep 24453

37. Leydolt C, Schartmuller D, Schwarzenbacher L, Schranz M, Schriefl S, Menapace R. Comparison of posterior capsule opacification development with 2 single-piece intraocular lens types. J Cataract Refract Surg. 2017;43:774-80.

38. Mencucci R, Favuzza E, Boccalini C, Gicquel JJ, Raimondi L. Square-edge intraocular lenses and epithelial lens cell proliferation: implications on posterior capsule opacification in an in vitro model. BMC Ophthalmol. 2015;15:5.

39. Hollick EJ, Spalton DJ, Ursell PG, Pande MV. Lens epithelial cell regression on the posterior capsule with different intraocular lens materials. Br J Ophthalmol. 1998;82:1182-8.

40. Sacu S, Findl O, Menapace R, Buehl W. Influence of optic edge design, optic material, and haptic design on capsular bend configuration. J Cataract Refract Surg. 2005;31:1888-94.

41. Menapace R, Schriefl S, Lwowski C, Leydolt C. Impact of primary posterior capsulorhexis on regeneratory after-cataract and
YAG laser rates with an acrylic micro-incision intraocular lens with plate haptics; 1-year and 3-year results. Acta Ophthalmol. 2019. https://doi.org/10.1111/aos.14156.

42. Vock L, Crnej A, Findl O, Neumayer T, Buehl W, Sacu S, et al. Posterior capsule opacification in silicon and hydrophobic acrylic intraocular lenses with sharp-edge optics six years after surgery. Am J Ophthalmol. 2009;147:683-90.

43. Sacu S, Menapace R, Findl O. Effect of optic material and haptic design on anterior capsule opacification and capsulorrhexis contraction. Am J Ophthalmol. 2006;141:488-93.

44. Sureshkumar J, Haripriya A, Muthukkaruppan V, Kaufman PL, Tian B. Cytoskeletal drugs prevent posterior capsular opacification in human lens capsule in vitro. Graefes Arch Clin Exp Ophthalmol. 2012;250:507-14.

45. D'Antin JC, Barraquer RI, Tresserra F, Michael R. Prevention of posterior capsule opacification through intracapsular hydrogen peroxide or distilled water treatment in human donor tissue. Sci Rep. 2018;24:12739-48.

46. Wormstone IM. Posterior capsule opacification: a cell biological perspective. Exp Eye Res. 2002;74:337-47.

47. Vock L, Georgopoulos M, Neumayer T, Buehl W, Findl O. Effect of the hydrophilicity of acrylic intraocular lens material and haptic angulation on anterior capsule opacification. Br J Ophthalmol. 2007;91:476-80. 